\title{
UNUSUAL METASTASES OF LUNG CANCER: BULBUS OCULI AND MAXILLARY SINUS
}

\author{
I. Ates ${ }^{1, *}$, O. Yazici ${ }^{1}$, H. Ates ${ }^{2}$, N. Ozdemir ${ }^{1}$, N. Zengin ${ }^{1}$ \\ ${ }^{1}$ Ankara Numune Education and Research Hospital, Department of Oncology, Ankara 06100, Turkey \\ ${ }^{2}$ Ankara Numune Education and Research Hospital, Department of Chest Disease, Ankara 06100, Turkey
}

Lung adenocarcinoma often makes metastasis to the brain, liver, kidneys, bone, bone marrow and adrenal glands. It can also make metastasis to other parts of the body rarely for example eye, nose, parotid gland and paranasal sinus. We did not encounter with combined ocular bulbus and the maxillary sinus metastases of lung cancer in the accessible literature. In this case report, a patient who was combined ocular bulbus and the maxillary sinus metastases of lung adenocarcinoma will be discussed.

Key Words: eye metastases, lung adenocarcinoma, paranasal sinus metastases.

Although lung adenocarcinoma often accompained metastasis to the brain, liver, kidneys, bone, bone marrow and adrenal glands, it can also make metastasis to other parts of the body rarely. Although very rarely seen lung cancer metastases were mentioned in the literature, we did not encounter with combined ocular bulbus and the maxillary sinus metastases. In this case report, a patient who was admitted with visual impairment and later on lung adenocarcinoma was diagnosed will be discussed.

\section{CASE REPORT}

Fifty-one-year-old male patient suffering from pain and blurred vision in the left eye admitted to ophthalmology outpatient clinic. In computerized tomography, two lesions $(10 \times 4 \mathrm{~mm}$ sized with light contrast enhancement at the posterior wall of the left bulbus oculi and $8 \mathrm{~mm}$ sized with peripheral contrast enhancement in the vicinity of the anterior wall of right maxillary sinus) were detected (Fig. 1).

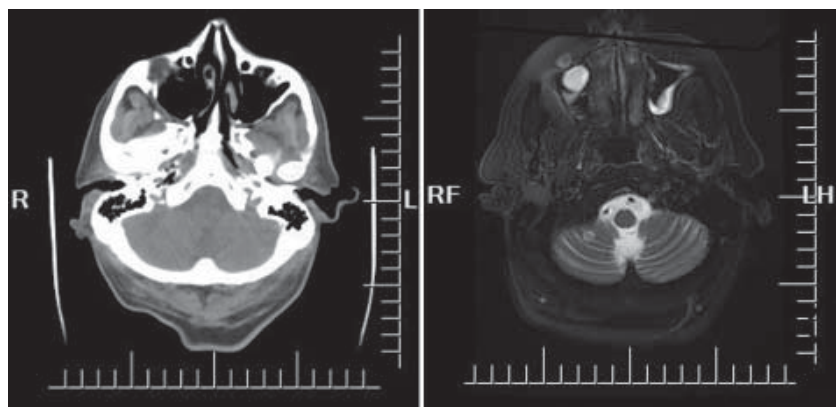

Fig. 1. In computerized tomography, $10 \times 4 \mathrm{~mm}$ sized tumor with light contrast enhancement at the posterior wall of the left bulbus oculi and $8 \mathrm{~mm}$ sized lesion with peripheral contrast enhancement in the vicinity of the anterior wall of right maxillary sinus

The patient was referred to the medical oncology outpatient clinic with the diagnosis of ocular bulbus and maxillary sinus metastases. In medical oncology outpatient clinic, we planned further examination to investigate the primary focus. Chest radiograph (Fig. 2) revealed irregular shaped lesion in middle lobe of the right lung. In thoracic computerized tomography, about $65 \times 59 \mathrm{~mm}$ sized, spicular contoured

Submitted: August 03, 2015.

*Correspondence: E-mail: dr.ihsanates@hotmail.com heterogeneous mass lesion with contrast enhacement that extending from the right hilar region to the middle lobe and collapsing the central lobe brochus was detected. Abdominal computerized tomography, upper and lower gastrointestinal endoscopy were normal. Maxillary sinus and eye metastases were confirmed with magnetic resonance imagining. Bronchoscopic biopsy with the preliminary diagnosis of lung cancer was reported as lung adenocarcinoma. Gemcitabine + cisplatin treatment was started.

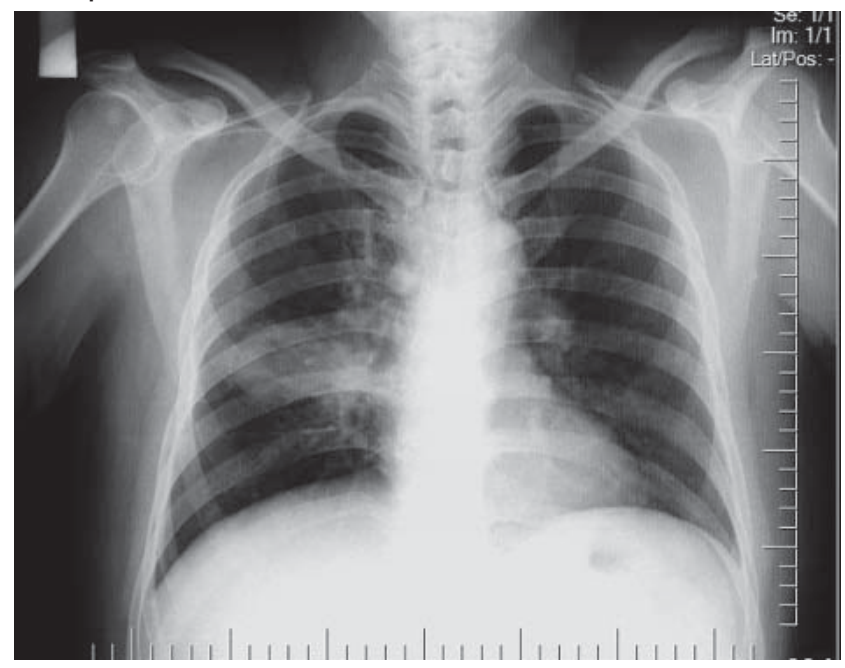

Fig. 2. Chest radiograph revealed irregular shaped lesion in middle lobe of the right lung

\section{DISCUSSION}

The most common malign and tumors of eye are metastatic tumors. In Shields and his colleagues study, most common metastatic tissues of eye have been reported as breast (47\%), lung (21\%), gastrointestinal $(4 \%)$, kidney $(2 \%)$, skin $(2 \%)$, prostate $(2 \%)$ and unknown primary (17\%) tissues [1]. Because the lymphatic system of the eye is weak, metastases to eye are generally with hematogenous way. The most frequently seen metastatic region is the choroid layer. Than metastases were seen in orbita, iris, ciliary body, optic nerve, conjunctiva, and eyelid, respectively. While $7-14 \%$ of patients is clinically asymptomatic, the rest aplies with pain, blurred vision, scotoma, metamorphopsia, photopsia, floaters [2]. Magnetic resonance imagining is the only diagnostic method that can easi- 
ly separate orbital metastasis from the other lesions of the bulbus. Metastatic lesion biopsy is not preferred because of tumor tissue seeding [3]. Radiotherapy is the treatment option when primary focus was not known. If focus was identified, systemic chemotherapy or hormonal treatment should be preferred [4].

Paranasal sinus metastases are seen rarely. It is seen 2 times more often in men than women but men predominance was seen in 5-6 decades, women predominance is seen in 6-7 decades. Patients usually present with headache, recurrent sinusitis and pain due to nerve involvement [4]. In the case series of Prescher and Brors that 169 patients were involved, maxillary sinus (33\%), sphenoid sinus (22\%), ethmoid sinus (14\%), frontal sinus (9\%) and multiple (22\%) sinus metastasis were observed [5]. The origins of metastasis were renal (40\%), lung (9\%), breast $(8 \%)$, prostate $(7 \%)$, tyroid $(7 \%)$, testicular $(5 \%)$, liver (4\%), respectively. In single lesions, as surgery was performed, it was seen that chemotherapy and radiotherapy efficancy were also effective.

We present this case because we did not encounter with combined ocular bulbus and maxillary sinus metastases due to lung adenocarcinoma in the literature. In our case, 51-years-old male patient was presented with eye findings. Metastatic lesions in ocular bulbus and maxillary sinus have been identified. Lung adenocarcinoma was detected in research of primer focus of this lesion. The patient has similar demographic characteristics and symptoms with the cases in the literature. Lesion in the maxillary sinus revealed as random.

As a result, when paranasal sinus or eyeball lesions are detected in cases with acute onset pathological vision problems and recurrent sinusitis, metastasis should be kept in mind. Investigation for the primary lesion must be done before the treatment.

\section{CONFLICT OF INTEREST}

The authors declare no conflict of interest.

\section{REFERENCES}

1. Shields CL, Shields JA, Gross NE, et al. Survey of 520 eyes with uveal metastases. Ophthalmology 1997; 104: 1265-76.

2. Wickremasinghe S, Dansingani KK, Tranos P, et al. Ocular presentations of breast cancer. Acta Ophthalmol Scand 2007; 85: 133-42.

3. Kaya A, Oner F, Fitoz S, et al. Metastatic lung cancer: presenting with ocular symptoms. Tuberk Toraks 2005; 53: 386-9.

4. Vega LG, Dipasquale J, Gutta R. Head and neck manifestations of distant carcinomas. Oral Maxillofac Surg Clin North Am 2008; 20: 609-23.

5. Prescher A, Brors D. Metastases to the paranasal sinuses: case report and review of the literature. Laryngorhinootologie 2001; 80: 583-94 (in German). 